a similar discontinuous spectrum by mixing together any or all of the bodies which alone could survive the aforesaid chemical treatment; - when all these facts are taken into consideration, and when due weight is given to the very characteristic spectrum reaction, I cannot help concluding that the most piobable explanation is that these anomalies are caused by the presence of an unknown body whose chemical reactions are not sufficiently marked to have enabled chemists to differentiate it from associated elements.

\section{THE DISTANCES OF THE STARS 1}

FVERY one who is acquainted with the rudiments of astronomy E knows that the sun with its attendant planets is merely an island group in the vast realms of space.

An island the size of this room in the middle of the Atlantic would hardly be more remotely apart from the surrounding sh rres than is our solar system from the bodies which surround it in space. To determine the distance from this solar system to the stars which surround it is the problem for our consideration to-night.

Recent Researches on 6r Cygni.-It is now almost exactly forty years (February 12, I84I) since the gold medal of the Royal
Astronomical Society was awarded to Bessel for his discovery of the annual parallax of 61 Cygni. On that occasion Sir John Herschel delivered an address, in which he glanced at the labours of Struve and of Henderson as well as those of Bessel. The discovery of the distances of the stars was alluded to as "the greatest and most glorious triumph which practical astronomy has ever witnessed." From this date the history of our accurate knowledge of the subject may be said to commence. Each succeeding race of astronomers takes occasion to investigate the parallax of 6r Cygni anew, with the view of confirming or of correcting the results arrived at by Bessel.

[The parallactic ellipse which the stars appear to describe, having been briefly explained, the method of dedncing the distances of the stars was pointed out.]

The attention of Bessel was directed to 6r Cygni by its proper motion of five seconds per annum. When Bessel undertook his labours in 1838 the pair of stars forming the double were in the position indicated on Fig. I. When O. Struve attacked the problem in 1853 the pair of stars forming 61 Cysni had moved considerably. Finally, when the star was observed at Dunsink in 1878 , it had made another advance in the same direction as before. In forty years this object had moved over an arc of the heavens upwards of three minutes in length.

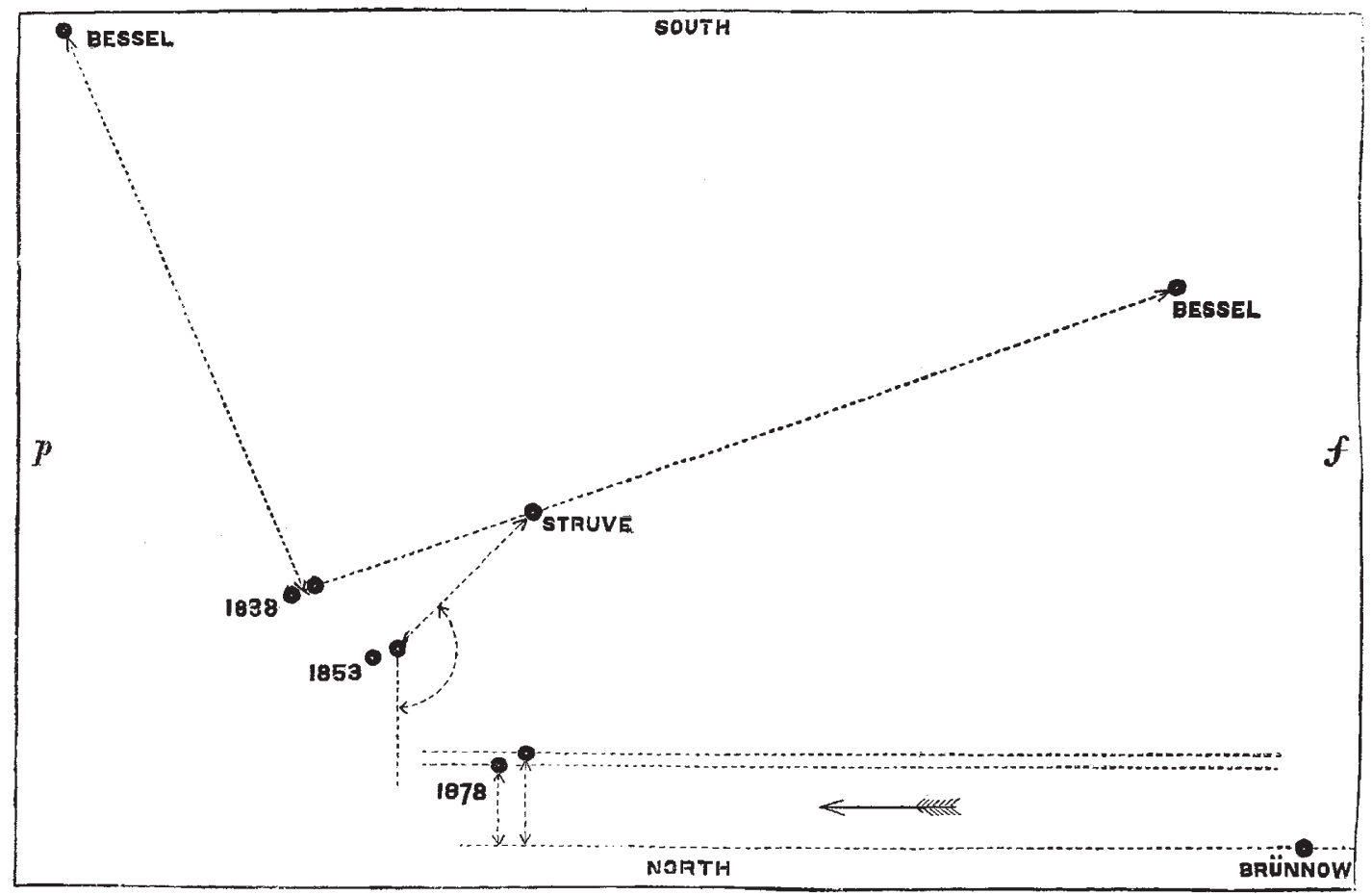

Fig. I. -6 r Cygni and parallax comparison stars.

The diagram contains four other stars besides the three position; of $6 \mathrm{r}$ Cygni. These are but small telescopic objects, they do not participate in the large proper motion of $6 \mathrm{I}$ Cygni, and they are undoubtedly much more remote from us. Bessel chose as the comparison stars the two objects marked with his name. $\mathrm{He}$ measured the distance from the central point of 6I Cygni to each of the two comparison stars. From a series of such measures he discovered the parallactic ellipse of $6 \mathrm{I}$ Cygni. He was led to the same ellipse by each of the two comparison star:

Fifteen years later (1853) Struve undertook a new determina tion. He chose a comparison star different from either of those Bessel had used. Struve's method of observing was also quite different from Bessel's. Struve made a series of measures of the distance and position of the comparison star from $6 \mathbf{I}(\mathrm{B})$ Cygni. Struve also succeeded in measuring the parallactic ellipse.

There was, however, an important difference between their

${ }^{x}$ Lecture at the Royal Institution of Great Britain, on Friday, February II, by Prof. Robert S. Ball, LL.D., F.R.S., Astron mmer Royal of Ireland. results. The distance, according to Bessel, was half as much again as Struve found. Bessel suid the distance was sixty billions of mile; ; Struva said it could not be more than forty billions.

The discrepancy may be due to the comparison stars. If Bessel's comparion stars were only about three times as far as 6r Cy yni, while Struve's star was about eight or ten times as far, the difference between Struve's results and Bessel's would be accounted for.

To settle the question, observation; were subsequently made by Auwers and others; the latest of these investigations is one which has recently been completed at Dunsink Observatory.

Dr. Brünnow proposed and indeed commenced a series of measures of the difference in declination between 61 Cygni and a fourth comparison star. These observations were made with the south equatorial at Dunsink. The carrying out of this work devolved on the lecturer, as Dr. Briinnow's successor. Two series of observations have been made, one with each of the components of $6 \mathrm{I}$ Cygni. The results agree very nearly with those of Struve. 
On a review of the whole question there seems no doubt that the annulal parallax of $6 \mathrm{r}$ Cygni is nearer to the half second found by Struve, than to the third of a second found by Bessel.

To exhibit the nature of the evidence which is available for the solution of such a problem, a diagram showing the second series of observations has been prepared (Fig. 2). The ab. scissæ are the dates of the second series of observations made at Dunsink. The ordinates indicate the observed effect of parallax on the difference of declinations between 6r (B) Cygni and the comparison star. Each dot represents the result of the observations made on the corresponding night. The curve indicates where the observations should have been with a parallax of $\mathrm{a}^{\prime \prime} \cdot 47$, the effect of the parallax in declination being only $0^{\prime \prime} 40$. The discordances are not so great as might perhaps be at first thought. The distance from the top of the curve to the horizontal line represents an angle of four-tenths of a second. This is about the apparent diameter of a pennypiece at the distance of ten miles. The discordance between the obserwations and the curve is in no case much more than half so great. It therefore appears that the greatest error we have made in these observations amounts to but two or three tenths of a second. This is equivalent to the error of pointing the telescope to the top edge of a penny-piece instead of to the bottom edge when the penny-piece was fifteen or twenty miles off.

The entire quantity to be measured is so small that the errors, minute as they are, bear a large proportion to the parallax. In this lies the weakness of such work. By sufficiently increasing the number of the observations, and by discussing them with the aid of the method of least squares, considerable confidence may be attached to the results.

Groombridge 1830.-This star bas been the subject of much parailax work. It has a telescopic proper mation of seven seconds annually. Mr. Huggins or Mr. Christie could perhaps ascertain by the spectroscope what its motion may be in the line of sight. From the theory of probabilities the total proper motion may not improbably be nine seconds. We shall however take it at seven seconds. The parallax has been determined by Struve and by Briinnow. It is very small, being one-tenth of a second. The actual velocity of 1830 Groombridge must therefore be at least 7o radii of the earth's orbit per annum, or 200 miles per second.

Newcomb has employed this result to throw light on the question as to whether all our stars form one system. If an isolated body in our system is to remain there for ever, the theory of gravitation imposes the imperative condition that the velocity of the body must not exceed a certain amount. Assuming that the stars are $100,000,000$ in number, and that each star is five times as large as the sun, assuming also that they are spread out in a layer of such dimensions that a ray of light takes 30,000 years to pass it, Newcomb shows that the critical velocity is twenty-five miles per second.

As this is only the eighth part of the velocity of Groombridge 1830, we are thus led to the dilemma that either the masses of the bodies in our system must be much greater than we have supposed, or Groombridge 1830 is a runaway star, which can never be controlled and brought back.

Search for Stars with Parallax. - The lecturer has been engaged for some years at Dunsink Observatory in a systematic search for stars which have an appreciable parallax. Up to the present about three hundred stars have been examined. In the majority of cases each of these stars has been observed only twice. The dates of the observations have been chosen so as to render the effects of parallax as manifest as possible. It is not of course expected that a small parallax of a few tenths of a second could be detected by this means. The errors of the observations would mask any parallax of this kind. It seems however certain that no parallax could have escaped detection if it equalled that of a Centauri, i.e. one second of arc.

The stars examined have been chosen on various grounds. It had been supposed that some of the red stars were possibly among the sun's neighbours, and consecuently many of the principal red stars were included in our list. No conspicuous parallax has however been detected in any of the red stars up to the present. Many of the principal double stars are also includect in the list. Other stars have been added on very various grounds; among them may be mentioned the Nova, which some time age burst out in the constellation Cyynus, and dwindled down again to a minute point. The earth's orbit however does not appear any larger when seen from Nova Cygni than from any of the other stars on our list.

Groombridge 1618. - We have however found one star which seems to have some claim to attention as one of the sun's neighbours. The star in question is Groombridge 1618. It lies in the constellation Leo, and is 6.8 magnitude. Groombridge 16.18 has a proper motion of $\mathrm{I}^{\prime \prime} 4$ annually. From a series of measurements of its distance made on fifty-five nights from a suitable comparison star the parallax of Groombridge 1618 appeared to be abont one-third of a second As this seemed to be a result of considerable interest, measures were renewed for a second series of forty nights. The result of the second series confirms the first. Measurements of the position angle were also made at the same time. Some difficulties not yet fully explained have arisen, but on the whole the measurements of the position angle seem to confirm the supposition that the parallax of Groombridge 16 I8 is about one-third of a

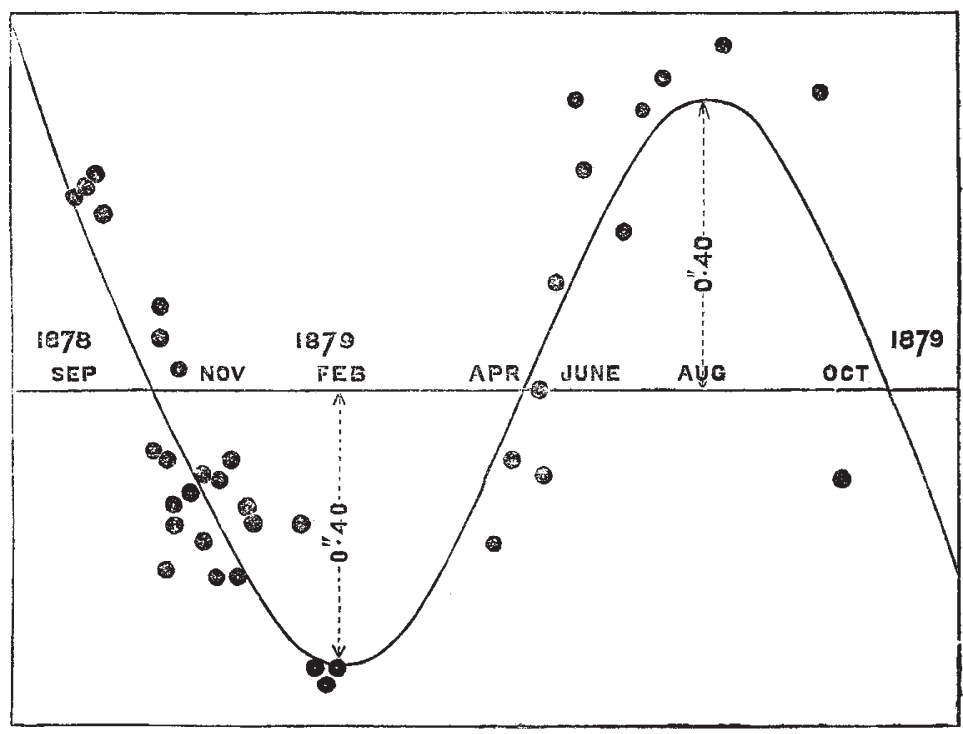
in declination of 6I (B) Cygni. Abscisse indicate
indicate parallax; dots indicate observations.

second. The orbit of the earth viewed from Groombridge 1618 is about the same size as a pennypiece at the distance of seven miles.

Secular Proper Motions.-Geologists have made us acquainted with the enormous intervals of time which have elapsed since the earth first became the abode of living animals. Regarding a period of $50,000,000$ of years as comparable with geologic time, some considerations were adduced as to the effect of proper motions during such an interval. It was pointed out that in all probability none of the stars now visible to the unaided eye can have then been visible from the earth.

The Nature of Space.-The possible connection of parallax work with the problems of the nature of space was then alluded to. It was shown that if space be hyperbolic the observed parallax is smaller than the true parallax, while the converse must be the case if space be elliptic. The largest triangle accessible to our measurements has for base a diameter of the earth's orbit, and for vertex a star. If the defect of the sum of the three angles of such a triangle from two riyht angles be in any case a measurable quantity, it would seem that it can only be elicited by observations of the same kind as those which are made use of in parallax investigations. 